\title{
The pupillary response to mental overload
}

\author{
MICHAEL JURIS and MANFRED VELDEN \\ Universität Mainz, 6500 Mainz, West Germany
}

\begin{abstract}
The hypothesis that the pupil constricts below base level in situations of mental overload was tested. Subjects had to perform in a four-alternative forced-choice task at $75 \%$, $100 \%$, and $125 \%$ the speed of their maximum processing capacity. No indication of a pupillary constriction in the overload situation was found. The pupil dilated under all three conditions. The pupil diameter of male subjects significantly decreased after the sharp increase at the beginning of the experimental phases, while the female subjects' pupil diameter remained at the same level after the initial increase. The amount of dilation depended on information load for male subjects only. Results were taken as further indication that no psychological stimulation may be specified that consistently elicits pupillary contriction.
\end{abstract}

Several physiological variables used in psychophysiological research have been assumed to be capable of making bidirectional changes, the direction of change depending on the kind of stimulation or the type of situational requirement. According to Lacey (1967), the heart rate decreases when the organism is taking in information from the environment, while it increases if disturbing or noxious stimulation is to be coped with. As has been proposed by Sokolov (1963) and confirmed by several western researchers (e.g., Hare, 1972), the constriction of the blood vessels in the head indicates a defensive response, while dilation is a component of the orienting response. A situational distinction partly analogous to Lacey's and Sokolov's has been made by Hess $(1965,1972)$ with respect to the pupillary response. He assumes that the pupil constricts as a response to unpleasant stimuli, while dilation is interpreted as an index of interest and/or positive affect. There has been considerable debate about this assumption. A review of the literature by Goldwater (1972) leaves doubt as to whether any psychological stimulation can be specified that reliably leads to pupillary constrictions. More recently, however, a further constriction-eliciting situation has been postulated. Poock (1973) and Poock and Noel (1975) proposed that information overload leads to a constriction of the pupil. In a four-alternative forced-choice task, Poock (1973) found a highly significant constriction of the pupil below a baseline level when stimuli were presented at $125 \%$ the speed of the subjects' maximum processing capacity. Poock and Noel (1975), stepwise increasing the presentation rate of the stimuli in the same type of experiment, observed that the

\footnotetext{
This article is based on data from the first author's doctoral dissertation. Requests for reprints should be sent to M. Juris, Psychologisches Institut des Universität, Abteilung Allgemeine und Experimentelle Psychologie, Saarstr. 21, 6500 Mainz, West Germany.
}

change from dilation to constriction occurred at a lower presentation rate for a noncompatible stimulusresponse arrangement as compared to a compatible one. Poock (1973) hypothesizes that the pupil constricts in order to inhibit reception of some of the input stimuli. In view of Hess' hypothesis, one might as well assume that the unpleasantness of the overload situation causes the pupil to constrict.

At the present stage of the discussion about systematic pupillary constrictions to psychological stimuli, however, it seems more profitable to further study the possibilities of such constrictions in general, rather than speculate about possible reasons for it. As has been suspected with respect to other psychological stimuli before (Loewenfeld, 1966), it could be possible that the constriction-eliciting effect of mental overload as demonstrated in the above two studies is artifactual. Blackwell, Hensel, and Sternthal (1970) have pointed out that a clear distinction must be made between a genuine constriction upon stimulus presentation and a return to base level after a dilation, which is also termed "constriction"' by some authors (e.g., Gardner, Beltramo, \& Krinsky, 1975). The assessment of the base level therefore requires special methodological care. A single base-level measurement at the beginning of the experiment, as was taken in the Poock and the Poock and Noel experiments, seems insufficient, because the baseline will probably drop due to a fall in activation level during the experiment. A baseline taken during the experiment or even values above it may therefore look like a constriction if compared to the baseline at the beginning. To make a more rigorous test of the overload hypothesis, an experiment was designed which had two decisive methodological characteristics: (1) A base level was measured before each of several phases of the experiment which differed in information load, and (2) the course of the pupillary response was traced second by second instead of taking means for the phases. Valuable information may be lost 
by averaging over time. To make a comparison of the data possible, the same type of task was used as in Poock's experiment.

\section{METHOD}

\section{Subjects}

Twenty-four subjects participated in the experiment, 12 of them high school students and 12 university students. There were 6 male and 6 female subjects in each of the groups.

\section{Apparatus}

Four red light emitting diodes were used for the stimulation. They were driven by a programmable electronic system. The intensity of the lights was so low as to be pupillomotorically inefficient. They were arranged in a horizontal line with a distance of $10 \mathrm{~mm}$ from each other and $98 \mathrm{~cm}$ from the subject. To avoid strong muscular activity when responding, micro switches were used as response keys that required only very light pressure for operation. The keys were installed such that two were to be operated by the left hand and two by the right hand. Subjects could adjust the position of the keys to their highest comfort.

For recording the pupillary movements, an apparatus very similar to the one described by Hess (1965), with head- and chinrest, was used, but instead of a film camera, an infrared TV system served for registration of the pupillary movement on a video tape. Measurement of the pupil diameter was done by using an electronic digital measuring device that has been described elsewhere (Juris, 1976). The pupillometer was placed in a sound-attenuated chamber; all other equipment was in a separate room.

\section{Procedure}

The subjects were instructed to respond to the lights by pressing the appropriate key, that is, the left one on the left hand if the far left light was lit, the right one on the left hand if the second light from the left was lit, and so on. The subjects were, furthermore, informed about a (fictitious) connection between intelligence and the ability to perform in the task. This was done in order to avoid subjects' giving up under the condition of information overload. In that case, a drop below the base level might occur which could not be unambiguously interpreted as actual constriction or reconstriction to the actual base level. The subjects were asked not to move the head during the whole experimental situation and to fixate the signal lights permanently, even during the pauses.

The experimental procedure itself was similar to the one described by Poock (1973). A first training phase with 40 stimuli and a pause of $20 \mathrm{sec}$ was succeeded by a phase in which the subjects had to respond as fast as possible. This phase also contained 40 stimuli. During these two phases, the signals changed automatically after each response, such that the individual maximum speed could be computed from the second phase. In the actual experimental phases, the presentation speed was set at $75 \%, 100 \%$, and $125 \%$ the individual maximum speed. The highly achievement-motivation-inducing general instruction (fictitious connection between intelligence and performance in the task) given before the training phase and the phase from which to compute the individual maximum speed was expected to ensure that the $125 \%$ condition would actually be an overload condition. The experimental phases lasted $20 \mathrm{sec}$ each, with 20 -sec pauses between them. The subjects were informed that the presentation speed would change from one experimental phase to the next. The order of the experimental phases was completely counterbalanced within each experimental group. The stimuli were given in random order.

\section{Data Reduction}

Pupil diameter was measured second by second during the whole experiment. The values for the treatment phases were ex- pressed as differences from the pretreatment base level which was defined as the mean for the $5 \mathrm{sec}$ preceding the treatment. Constrictions of the pupil would therefore yield negative values, dilations, positive values. The difference scores were furthermore range corrected analogous to the method proposed by Lykken, Rose, Luther, and Maley (1966). For this purpose, each difference score was divided by the difference between the greatest and the smallest value of the respective individual. Statistical analysis of the difference scores $(\Delta \phi)$ as well as of the range corrected difference scores $(\Delta \phi)$ was made by means of an analysis of variance with factors Sex, Information Load, and Time (seconds), the latter two implying repeated measurements and thus correlated scores. Previous analysis had shown that the distinction between high school and university students was irrelevant because this factor showed niether a significant main effect nor a significant interaction with any of the other factors (each $F<1.00$ ).

\section{RESULTS}

Under the $75 \%, 100 \%$, and $125 \%$ speed conditions, there were $81 \%, 53 \%$, and $32 \%$ correct responses, indicating that mental overload had actually occurred.

Figure 1 shows the pupillary response of the male and female subjects averaged over the three experimental phases. There is a pronounced dilatory change of the pupil diameter over time which is statistically highly significant $[F(19,418)=10.95, p<.01]$. The slight constriction in the 1st second we see for the female group is probably due to accommodation, which means that the subjects deviated from the instruction to fixate the display during the pauses, too. The discrepancy between the trends of the two groups is statistically reflected by a highly significant Time by Sex interaction $[F(19,418)=2.73, p<.01]$. The pupil diameter of the male subjects starts to drop from about the 6th second on, while the females' remains nearly constant after the initial sharp increase. Even though there is no significant main effect of the

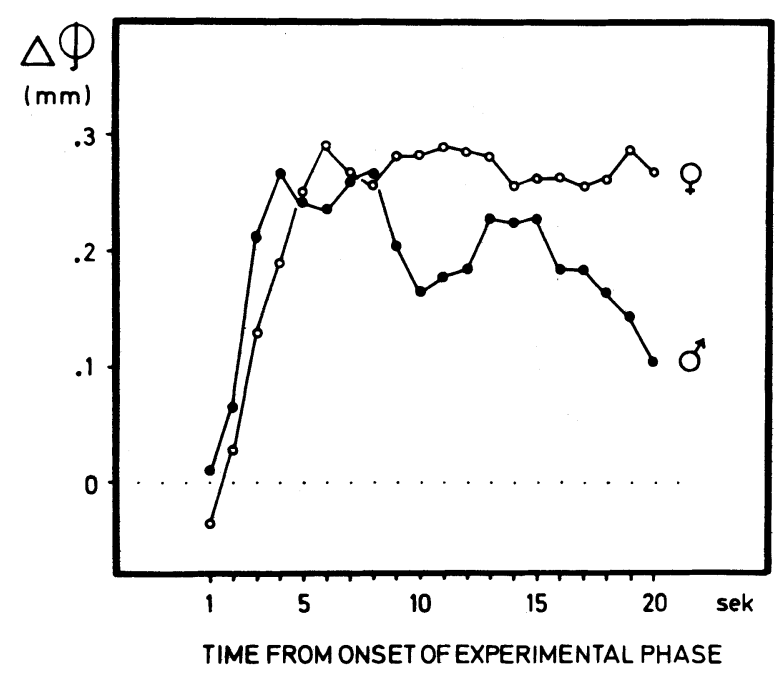

Figure 1. Change of pupil diameter averaged over the three experimental phases for male and female subjects. Difference from prephase level. 

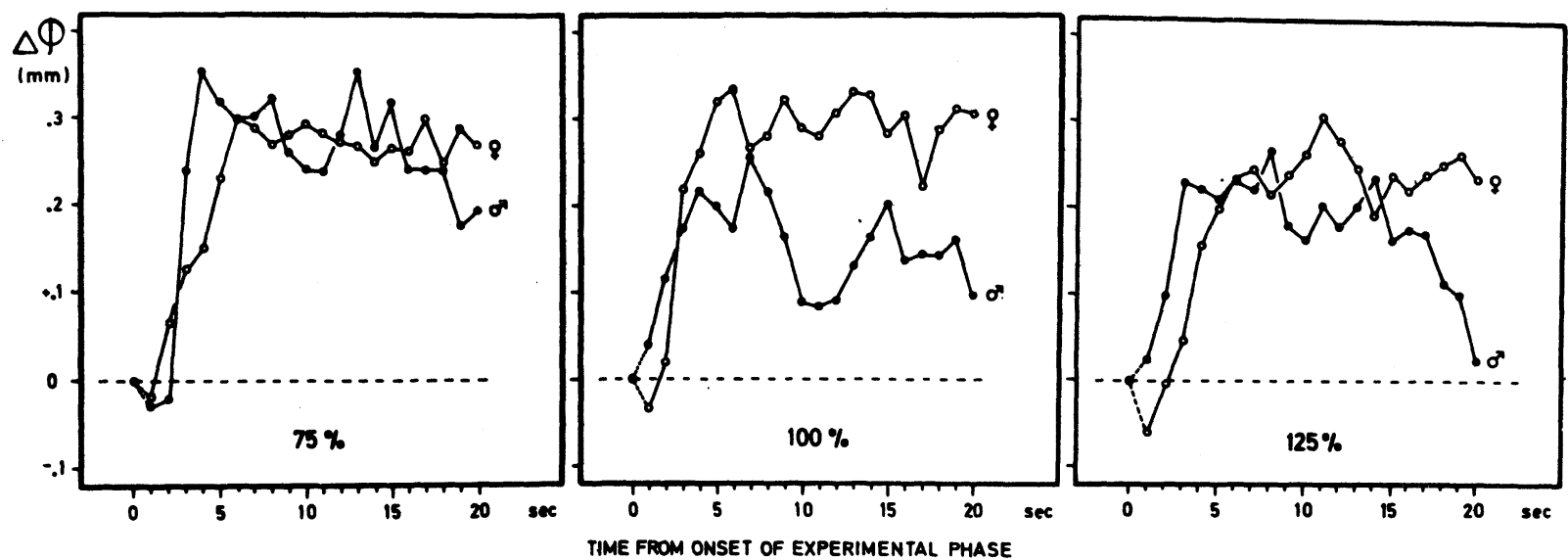

Figure 2. Change of pupil diameter in the three experimental phases for male and female subjects. Difference from prephase level.

experimental treatments $(\mathrm{F}<1.00)$ and no significant Time by Sex by Information Load interaction $\left[F(38,836)=1.36, F_{5 \%}=1.42\right]$, a graphic presentation of the responses of the two groups to each of the three information load phases yields some additional information (Figure 2).

While the females show essentially the same response under all three information load conditions, the male subjects' drop in pupil diameter over time is especially pronounced under the $125 \%$ condition, where it goes down almost to the prephase level. It looks as if the male subjects do not try hard till the end of the experimental phase under the overload condition. There is no indication, however, for a constriction of the pupil to any of the three experimental conditions.

Range correction of the difference scores enhanced the already very significant $F$ values for the factor of Time as well as the Time by Sex interaction $(F=13.89$ and $F=5.15$, respectively) and also resulted in a highly significant Sex by Information Load interaction $[\mathrm{F}(2,44)=5.19, \mathrm{p}<.01]$ which had been insignificant for the nontransformed difference scores $(F<1.00)$. Figure 3 shows the dependence of the mean change in pupil diameter from information load for the two groups when range-corrected scores are considered. A dependence seems to exist for the male but not for the female subjects. This picture could not be expected from the simple difference scores that we see in Figure 2. The male subjects' effort under the $100 \%$ and $125 \%$ conditions is obviously higher than the untransformed difference scores would make one believe. A tendency of the pupil diameter to increase with information load for range corrected scores $\left[\mathrm{F}(2,44)=2.80, \mathrm{~F}_{5 \%}=\right.$ 3.21], which appears if the two experimental groups are being pooled, is obviously misleading due to the totally different picture for these two groups.

\section{DISCUSSION}

In view of the bulk of experimental evidence (Goldwater, 1972), our results may be interpreted as some additional indication that no psychological stimulation or situation can be described to date that consistently leads to pupillary constrictions that are not reconstrictions after a dilation. Studies reporting constrictions to particular stimuli with particular subjects (Libby, Lacey, \& Lacey, 1973; Polt \& Hess, 1968) are not persuasive unless the relevant individual difference factors and stimulus parameters can be identified. Careful assessment of the base level as well as complete tracing of the pupillary movement over time are necessary when the capability of the pupil to constrict to psychological stimuli is being tested.

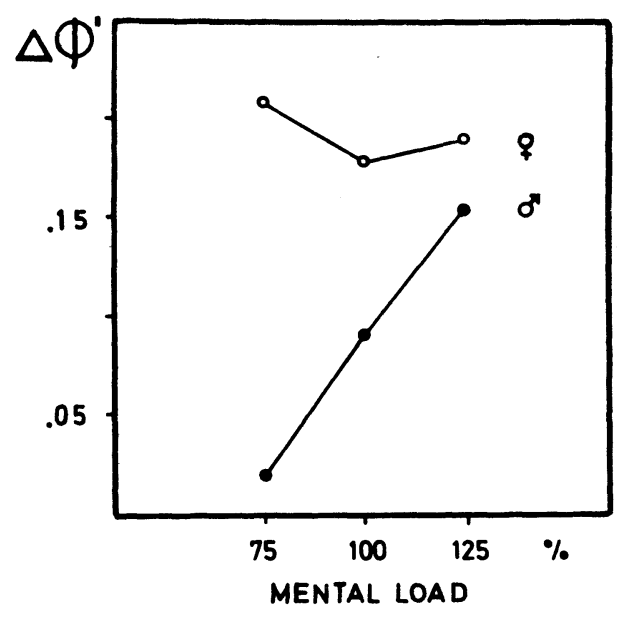

Figure 3. Influence of information load on pupil diameter for male and female subjects averaged over seconds. Rangecorrected difference scores. 
As has been reported by other researchers before (Polt \& Hess, 1968), the sex factor seems to be a significant determinant of pupillary movements. This may be taken as a warning not to make statements about pupillary responses to psychological stimuli in a general, group-unspecific form. Moreover, the study gives additional empirical support to the significance of the range correction, a good theoretical basis for which has been elaborated by Lykken (1968) and Lykken and Venables (1971).

\section{REFERENCES}

Blackwell, R. D., Hensel, J. S., \& Sternthal, B. Pupil dilation: What does it measure? Journal of Advertising Research. 1970, 10, 15-18.

Gardner, R. M., Beltramo, J. S., \& Krinsky, R. Pupillary changes during encoding, storage, and retrieval of information. Perceptual and Motor Skills, 1975, 41, 951-955.

Goldwater, B. C. Psychologic significance of pupillary movements. Psychological Bulletin, 1972, 77, 340-355.

HARE, R. D. Cardiovascular components of orienting and defensive responses. Psychophysiology, 1972, 9. 606-614.

Hess. E. H. Attitude and pupil size. Scientific American, 1965, 212, 46-54.

Hess, E. H. Pupillometrics. A method of studying mental. emotional, and sensory processes. In N. S. Greenfield \& R. A. Sternbach (Eds.), Handbook of psychophysiology. New York: Holt, Rinehart, \& Winston, 1972.
JURIS, M. A faster and more accurate instrument for digital measurement of pupil diameter. Psychophysiology, 1976, 13. 363-365.

LACEY, J. I. Somatic response patterning and stress: Some revisions of activation theory. In M. H. Appley \& R. Trumbull (Eds.), Psychological stress. New York: Appleton-Century-Crofts. 1967.

LiB BY, W. L., LACEY, B. C., \& LACEY, J. I. Pupillary and cardiac activity during visual attention. Psychophysiology, 1973, 10. 270-294.

Loewenfeld, I. E. Comment on Hess' findings. Survey of Ophthalmology, 1966, 11, 293-294.

LYKKEN. D. T. Neuropsychology and psychophysiology. In E. F. Borgatta \& E. W. Lambert (Eds.), Handbook of personality research. Chicago: Rand McNally, 1968.

Lykken, D. T.. Rose, R., Luther, B., \& Maley, M. Correcting psychophysiological measures for individual differences in range. Psychological Bulletin, 1966, 66. 481-484.

LyKKEN, D. T., \& Venables, P. H. Direct measurement of skin conductance: A proposal for standardization. Psychophysiology, 1971, 8, 656-672.

Polt, J., \& Hess, E. H. Changes in pupil size to visually presented words. Psychonomic Science, 1968, 12. 389-390.

Poock. G. K. Information processing versus pupil diameter. Perceptual and Motor Skills, 1973, 37, 1000-1002.

Poock, G. K., \& Noel, C. E. Effects of layout of workplace on pupillary diameter. Perceptual and Motor Skills, 1975, 40. 304.

Sokolov, E. N. Perception and the conditioned reflex. Oxford: Pergamon Press, 1963.

(Received for publication April 4, 1977; accepted June 28. 1977.) 